Our deepest thanks are owed to the medical and nursing staff of the unit where this study was carried out, who so generously enabled the work to succeed. In particular we thank Drs Sylvia Blunden, Hugh Baker, Alan Black, Stephen Frank, and Peter Dally. Thanks are due to Leonie Cox, who met the secretarial and organisational needs of the project; Professor John Wing, who provided the computer analysis of the PSE data; and Ken MacRae, who provided statistical advice and computer facilities at Charing Cross Medical School. The study was supported by a grant from the DHSS.

Requests for reprints and copies of the Social Behaviour Assessment Schedule should be addressed to: Professor S R Hirsch, Department of Psychiatry, Charing Cross Hospital, Fulham Palace Road, London W6 8RF.

\section{References}

1 Glick, I D, Hargreaves, W A, and Goldfield, W N, Archives of General Psychiatry, 1974, 30, 3630.

${ }^{2}$ Herz, M I, Endicott, J, and Spitzer, K L, American fournal of Psychiatry, 1977, 134, 502.

${ }^{3}$ Platt, S, et al, Social Behaviour Assessment Schedule (SBAS). I : Development and Contents of a New Interview Schedule. Submitted for publication.

Platt, S, et al, Social Behaviour Assessment Schedule (SBAS). II : Reliability of a New Interview Schedule. To be published.

5 Wing, J, personal communication.

6 Jones, R J, and Goldberg, D. To be published.

(Accepted 27 November 1978)

\title{
Gentamicin- and silver-resistant pseudomonas in a burns unit
}

\author{
K BRIDGES, A KIDSON, E J L LOWBURY, M D WILKINS
}

British Medical fournal, 1979, 1, 446-449

\section{Summary and conclusions}

In 1977-8 gentamicin-resistant strains of Pseudomonas aeruginosa became very common in a burns unit, over $90 \%$ being resistant at the peak of the outbreak. Some strains were also resistant to silver nitrate, though silver resistance was not found in any other strains of Ps aeruginosa isolated. Unlike the gentamicin resistance, the silver resistance was unstable, and strains became sensitive on repeated subculture. All the gentamicinresistant strains of $P s$ aeruginosa were of the same serotype (O:11, H:2,5). Though gentamicin resistance could be transferred in vitro from resistant strains of Ps aeruginosa to one sensitive strain of Ps aeruginosa, there was no evidence of in-vivo transfer of gentamicin resistance between strains of pseudomonas in the patients' burns, nor was there evidence of transfer of gentamicin resistance between Ps aeruginosa and enterobacteria. Carbenicillin-resistant and gentamicin-resistant Ps aeruginosa were sometimes found in the same burns, but no gentamicin-carbenicillin (doubly) resistant strains were found among the 986 strains tested during the outbreak.

The outbreak of gentamicin-resistant Ps aeruginosa from burns was not reduced by stopping treatment with gentamicin and its analogues but only by segregating all patients with Ps aeruginosa in one of the two wards of the unit and admitting new patients only to the other ward.

\section{Introduction}

Systemic gentamicin and topical silver preparations have been used in this unit since the mid-1960s. For most of that period few strains of Pseudomonas aeruginosa isolated from the patients'

\footnotetext{
MRC Burns and Industrial Injuries Unit, Birmingham Accident Hospital, Birmingham

K BRIDGES, BSC, microbiologist

A KIDSON, FIMIs, chief medical laboratory scientist

E J L LOWBURY, DM, FRCP, bacteriologist, visiting professor of medical microbiology, University of Aston, Birmingham

M D WILKINS, FIMLS, senior research officer
}

burns have been resistant to gentamicin and none have been resistant to silver. ${ }^{-3}$ In 1977 , however, gentamicin resistance became very common, and the resistant strains were often also resistant to silver nitrate. Since gentamicin (and its analogues) and silver preparations are probably the most effective of the few agents available for, respectively, systemic treatment and topical prophylaxis against pseudomonal infections, the emergence of these resistant variants posed a serious threat to controlling infection in the unit.

We describe here the emergence of these resistant variants and our measures to eliminate them. We also describe in-vitro tests for transfer of resistance.

\section{Incidence and control of resistance}

METHODS

Burns were examined for bacterial flora when patients were admitted to the unit, at all changes of dressings, at operations, and daily if the burns were exposed, by methods described elsewhere. ${ }^{2}$ Ps aeruginosa, Escherichia coli, Klebsiella spp, Enterobacter spp, Proteus spp, and other Gram-negative bacilli were identified by standard methods. ${ }^{4}$ All isolates of Gram-negative bacilli were tested for sensitivity to gentamicin, amikacin, carbenicillin, neomycin, trimethoprim, and sulphadiazine by a ditch-plate diffusion test. ${ }^{5}$ Tube dilution tests were also made on some of the strains, using nutrient broth as the culture medium, with 1 drop $(0.02 \mathrm{ml})$ of a $1 / 1000$ dilution of an overnight broth culture as the inoculum. In the ditchplate test for gentamicin sensitivity two dilutions were used, one containing $50 \mathrm{mg} / \mathrm{l}$ and one containing $10 \mathrm{mg} / \mathrm{l}$ of gentaruicin sulphate in the nutrient agar of the ditch. Strains were considered sensitive if the minimal inhibitory concentration (MIC) of gentamicin was $8 \mathrm{mg} / \mathrm{l}$ or less. All strains which were gentamicin-sensitive by the tube dilution test showed zones of inhibition in the ditch-plate diffusion test which were closely similar to that obtained with the sensitive control strain (MIC $2 \mathrm{mg} / \mathrm{l}$ ) tested on the same plate; resistant strains, which had MICs of $16-32 \mathrm{mg} / \mathrm{l}$, grew across the agar of the ditch containing $10 \mathrm{mg} / \mathrm{l}$ gentamicin.

Tests for silver nitrate resistance were performed as described by Cason et al, ${ }^{6}$ using a plate dilution method with serial dilutions of silver nitrate in nutrient agar.

\section{Results}

Gentamicin-resistant Ps aeruginosa

Table I shows the proportion of gentamicin-resistant strains of Ps aeruginosa isolated from burns from June 1977 to September 1978. Each patient had one or more burns, and each burn colonised by 
TABLE I-Gentamicin-resistant Ps aeruginosa isolated from burns in 1977-8

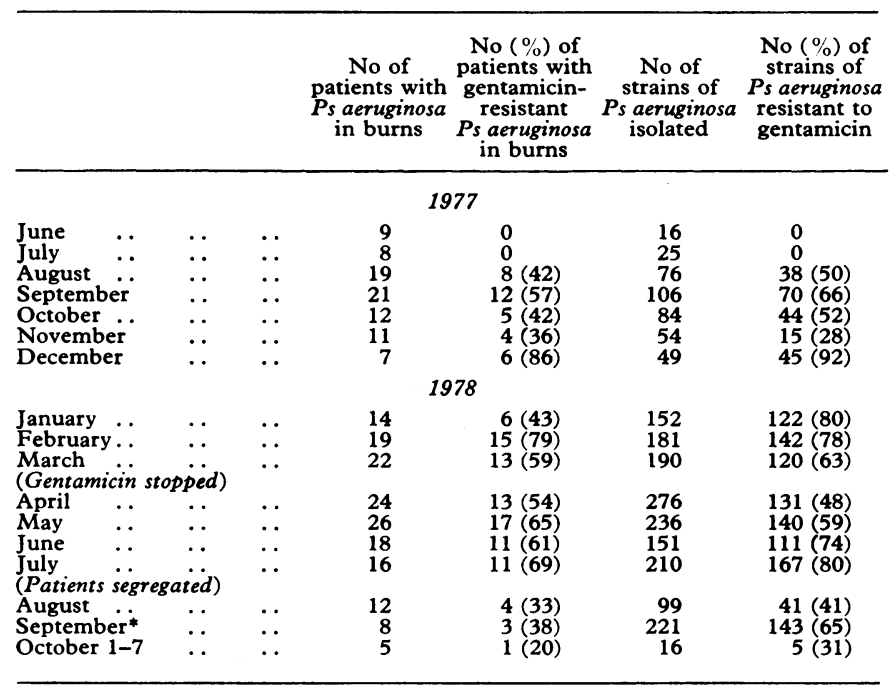

*Increased isolations in September followed treatment of one patient with gentamicin.

TABLE II-Gentamicin-resistant "coliform bacilli"* isolated from burns in the period when gentamicin-resistance in Ps aeruginosa was common

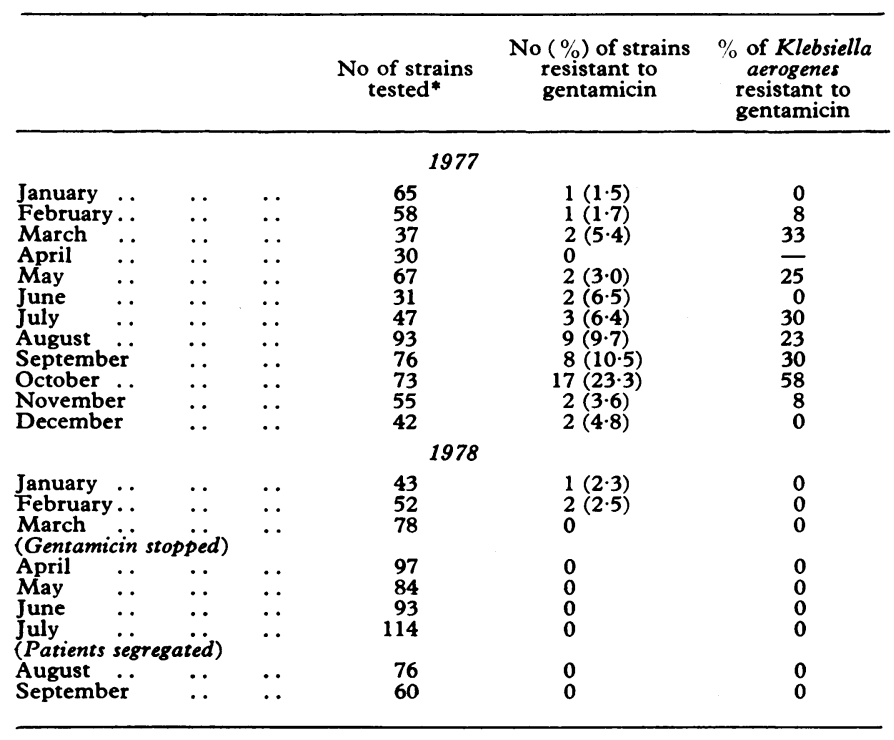

*Strains tested (and No and $\%$ resistant to gentamicin): Klebsiella aerogenes 210
$(39 ; 19 \%)$, Proteus spp $385(0), A$ anitratum $278(9 ; 3 \%)$, others $498(4 ; 0.8 \%)$.

Ps aeruginosa yielded the organism on one or more successive swab samplings. The incidence of gentamicin-resistant isolates rose rapidly to $50 \%$, and then to over $90 \%$ in December 1977 and remained high for about a year.

Some gentamicin-resistant and gentamicin-sensitive strains of Ps aeruginosa were phage-typed and serotyped at the Central Public Health Laboratory, Colindale. All the gentamicin-resistant strains were of one serotype $(\mathrm{O}: 11, \mathrm{H}: 2,5)$ and untypable by phages sensitive strains were all of other serotypes, the commonest being type $\mathrm{O}: 16, \mathrm{H}: 4$.

We tried to eliminate the gentamicin-resistant strains from the unit by discontinuing gentamicin and tobramycin treatment in patients with severe pseudomonal infection on 13 March 1978 and using amikacin, which was fully active against all the gentamicin-resistant strains, instead. This did not reduce the incidence of gentamicinresistant Ps aeruginosa. On 15 July 1978 we segregated all patients with gentamicin-resistant pseudomonal infections or infections with $P s$ aeruginosa of unknown sensitivity in one of the two wards of the burns unit, and closed that ward to further admissions until all the patients in it with resistant pseudomonads had been discharged. The two wards had separate nursing staffs, so that Ps aeruginosa was not likely to be transferred from one to the other. The numbers of patients carrying gentamicin-resistant (and other) Ps aeruginosa in the unit fell progressively after segregation (table I). The increase in the proportion of gentamicin-resistant isolates in September 1978, when they were thought to have been eliminated, was due to the continued presence of two patients with extensive burns, one of whom had sensitive Ps aeruginosa in his burns which were replaced by the resistant strain of Ps aeruginosa when he was treated with gentamicin; this infection was transferred to the other extensively burnt patient by crossinfection.

Table II shows the proportion of gentamicin-resistant isolates of enterobacteria (predominantly Klebsiella spp, Escherichia coli, Proteus spp, and Acinetobacter anitratum) in a sampling of one strain per patient per week from burns, and the proportion of patients with these organisms in whom the strains were resistant to gentamicin from January 1977 to September 1978. There were few gentamicinresistant coliform bacilli and no gentamicin-resistant Proteus spp. Klebsiella spp showed some resistance, with a temporary increase in October 1977. The frequency of isolation of gentamicin-resistant enterobacteria fell several weeks before the use of gentamicin and tobramycin in the ward was stopped; their presence was apparently unrelated to the presence of gentamicin-resistant Ps aeruginosa, which persisted for long after the gentamicin-resistant enterobacteria had become scarce. Gentamicin-resistance in Ps aeruginosa rose to its highest in December 1977, when gentamicin-resistant Klebsiella had virtually disappeared from the unit.

\section{Silver-resistant Ps aeruginosa}

Strains of Ps aeruginosa isolated in this unit have been tested for sensitivity to silver nitrate since 1965 , but no resistant strains were found until August 1977. Only strains of serotype $\mathrm{O}: 11, \mathrm{H}: 2,5$ (which were also resistant to gentamicin) were resistant to silver, but they lost their silver resistance on repeated subculture, while retaining their gentamicin resistance.

\section{Carbenicillin-resistant Ps aeruginosa}

After Ps aeruginosa carrying plasmids determining high-level resistance to carbenicillin had been eliminated from the unit in 1972, 7 such strains were not seen again until October 1977, when highly resistant strains producing diffusible "carbenicillinase" reappeared. These carbenicillin-resistant strains were all of serotypes other than type $\mathrm{O}: 11, \mathrm{H}: 2,5$. On several occasions and in several patients two strains of Ps aeruginosa, one resistant to gentamicin and one highly resistant to carbenicillin, were present simultaneously in the same burn, but no strains resistant to both gentamicin and carbenicillin were found in these burns or among 986 strains of Ps aeruginosa tested while both gentamicin and carbenicillin resistant strains were present in the unit.

\section{Experiments on resistance transfer}

Several studies were made to discover whether the gentamicin and carbenicillin resistance of the strains of Ps aeruginosa and of enterobacteria could be transferred on resistance plasmids to other bacteria of the same or different species.

\section{METHODS}

We used the same technique of testing for resistance transfer as in earlier studies on sulphonamide resistance ${ }^{8}$ though we modified it to suit the sensitivity and resistance patterns of the donor and recipient strains under investigation. One-ml amounts of 24-hour nutrient broth culture of donor and recipient strains were added to $2 \mathrm{ml}$ of nutrient broth, and the mixed culture was incubated at $37^{\circ} \mathrm{C}$ for 24 hours. It was then centrifuged at $3000 \mathrm{rpm}$ for 15 minutes, and the deposit was resuspended in $0.02 \mathrm{ml}$ of nutriert broth. This was plated on nutrient agar containing appropriate concentrations of antibiotics to inhibit the growth of the donor strain (see below). After incubation at $37^{\circ} \mathrm{C}$ for 24 hours the plates were examined and the colonics were picked, identified by routine methods, tested for sensitivity to gentamicin and carbenicillin by methods described above, and tested for serotype and phage type at the Central Public Health Laboratory, Colindale.

We performed a test to see whether gentamicin resistance could be transferred from Ps aeruginosa to a culture of $E$ coli $\mathrm{K} 12$ which was 
resistant to $3000 \mathrm{mg} / \mathrm{l}$ of streptomycin. The selective medium contained $3000 \mathrm{mg} / \mathrm{l}$ streptomycin and $12 \mathrm{mg} / \mathrm{l}$ gentamicin. The $E$ coli, if resistant to gentamicin, would grow well on this medium, and the $P$ s aeruginosa appeared as very small colonies. Tests were made on six isolates of gentamicin-resistant $P s$ aeruginosa. As an alternative method tests (on eight isolates of gentamicin-resistant Ps aeruginosa) were done with nutrient agar plates containing $12 \mathrm{mg} / \mathrm{l}$ gentamicin and using anaerobic culture to inhibit the growth of the pseudomonas. Twelve tests were also set up on plates of MacConkey's medium containing $12 \mathrm{mg} / 1$ gentamicin.

For testing the transfer of resistance plasmids from gentamicinresistant to gentamicin-sensitive strains of Ps aeruginosa, recipient strains resistant to carbenicillin (MIC $>4000 \mathrm{mg} / \mathrm{l}$ ) were used, and the selective medium of the plates contained $300 \mathrm{mg} / \mathrm{l}$ carbenicillin and $16 \mathrm{mg} / 1$ gentamicin. This inhibited both donor and recipient strains but would allow growth of either a recipient that had acquired gentamicin resistance by transfer of a plasmid from the donor or of a presumptive donor of gentamicin resistance that had acquired carbenicillin resistance from the presumptive recipient of gentamicin resistance. Thirty-four tests were made, including tests using different proportions of donor to recipient strains $(1: 2,1: 5$, and $1: 10)$.

The transfer of gentamicin resistance from a resistant Klebsiella aerogenes to a sensitive strain of $P s$ aeruginosa (Ellsworth) was also tested; the klebsiella was isolated from a burn wound which also contained gentamicin-resistant Ps aeruginosa. Five donor isolates were tested for transfer of resistance by plating the mixture of donor and recipient strains on a selective medium containing $16 \mathrm{mg} / \mathrm{l}$ gentamicin and $200 \mathrm{mg} / \mathrm{l}$ neomycin (which would inhibit the klebsiella but not a gentamicin-resistant pseudomonas). Tests were also made to detect possible transfer of gentamicin resistance from a resistant $P$ s aeruginosa to a sensitive klebsiella strain isolated from the same patient.

Tests for transfer of carbenicillin resistance from $P s$ aeruginosa to $E$ coli $\mathrm{K} 12$ were made in the manner described by Roe et al ${ }^{\circ}$ but with selective plates containing $3000 \mathrm{mg} / 1$ streptomycin and $200 \mathrm{mg} / \mathrm{l}$ carbenicillin; the higher concentration of streptomycin was used because the carbenicillin-resistant $P_{s}$ aeruginosa was moderately resistant to streptomycin. As an alternative technique, plates containing $200 \mathrm{mg} / 1$ carbenicillin were incubated anaerobically. Seven isolates of carbenicillin-resistant Ps aeruginosa were tested.

\section{RESULTS}

Tests for transfer of gentamicin-resistance from 26 donor strains of $P s$ aeruginosa to $E$ coli $\mathrm{K} 12$ gave negative results. The results of transfer testing were assessed by aerobic culture on streptomycingentamicin agar plates for six of the donor strains, by anaerobic culture on gentamicin agar for eighi, and by aerobic culture on gentamicin-MacConkey plates for 12 .

Tests for transfer of gentamicin resistance from one strain of $P_{s}$ aeruginosa to another of the same species were negative with 26 donor strains, using six gentamicin-sensitive recipient strains, four of them mated each with two donor strains, one (1686) with eight donor strains, and one (7188B) with 10 donor strains. With the gentamicin-sensitive strain 8005 (serotype $\mathrm{O}: 16, \mathrm{H}: 4$ ) five of the donor strains $(6005,6189,6320,6369$, and 6332$)$ which had failed to transfer gentamicin resistance to recipient strain 1686 transferred gentamicin resistance. Growth of these organisms on the selective medium may have been due either to the transfer of gentamicinresistance from the donor strains or to transfer, in the opposite direction, of carbenicillin-resistance from the supposed recipient strain To clarify this point the strains were serotyped. All five of the doubly resistant strains growing on the selective medium were found to be of serotype $\mathrm{O}: 16, \mathrm{H}: 4$. We therefore concluded that gentamicin resistance had been transferred from the presumptive donor of gentamicinresistance to strain 8005 . Carbenicillin resistance was not transferred from $P$ s aeruginosa to $E$ coli $\mathrm{K} 12$ or to other strains of $P$ s aeruginosa.

\section{Discussion}

The emergence and subsequent predominance of gentamicinresistant $P$ s aeruginosa in many burn wounds has been described by Stone, ${ }^{10}$ and resistant strains were often isolated in some centres. ${ }^{11}{ }^{12}$ Witchitz and Chabbert ${ }^{13}$ and Kontomichalou et al ${ }^{12}$ have described the intraspecies transfer of gentamicin resistance plasmids between different strains of $P s$ aeruginosa and also intergeneric transfer of such plasmids.
Our studies have shown the rapid emergence and continued presence of a high incidence of gentamicin-resistant $P s$ aeruginos $a$ in 1977-8. All the gentamicin-resistant strains were of one serotype $(\mathrm{O}: 11, \mathrm{H}: 2,5)$, though sensitive strains of other serotypes were present in the unit and were several times found in the same burn. During the same period a larger proportion of gentamicin-resistant strains of enterobacteria (mainly Klebsiella spp) briefly emerged, but the resistance disappeared long before the introduction of measures that reduced the incidence of gentamicin-resistant $P$ s aeruginosa in the wards. Such results contrasted with our findings when transferable carbenicillinresistance became predominant among the strains of $P_{s}$ aeruginosa isolated in this unit. ${ }^{1}$ At that time several different phage or serotypes of Ps aeruginosa showed plasmid-determined carbenicillin-resistance. Plasmid-determined resistance of enterobacteria to tetracycline, ampicillin, kanamycin, and cephaloridine increased in parallel and persisted until all the relevant antibiotics were discontinued. ${ }^{714}$ By contrast, the gentamicin resistance of Ps aeruginosa which predominated in 1977-8 was apparently not transferred in the burns either to sensitive strains of Ps aeruginosa or to enterobacteria, though there was evidence of in-vitro transfer of gentamicin-resistance from the endemic strain to one of the recipient strains used in transfer experiments. Unlike the carbenicillin-resistant strains of $P$ s aeruginosa which were predominant in 1969-72, and which quickly disappeared from the unit when the use of carbenicillin was stopped, gentamicin-resistant $P$ s aeruginosa persisted after withdrawal of gentamicin and were eliminated only by segregating patients carrying such strains. Measures that eliminate one pattern of resistance will not necessarily eliminate another pattern. Even a multiresistance plasmid may sometimes be eliminated from a ward by withdrawing only one of the antibiotics for which the plasmid codes resistance. ${ }^{8}$

In-vitro tests showed that gentamicin-resistance was not transferred from $P$ s aeruginosa to $E$ coli $\mathrm{K} 12$ and not usually transferred from one strain of Ps aeruginosa to another. A gentamicin-resistant klebsiella, present in burns which were also colonised by a gentamicin-resistant Ps aeruginosa, also did not transfer this resistance in vitro to a gentamicin-sensitive $P s$ aeruginosa. Only one of the gentamicin-sensitive strains of $P_{s}$ aeruginosa tested as recipients accepted the plasmid (becoming doubly resistant to carbenicillin and gentamicin and retaining the serotype of the carbenicillin-resistant recipient).

Silver nitrate resistance was associated with gentamicin resistance in several strains and not found in any gentamicinsensitive strains of Ps aeruginosa. But the gentamicin resistance persisted, while silver resistance was readily lost on storage or subculture of these strains, showing that there was no genetic link between these resistance characters. The relevance of silver resistance in relation to topical prophylaxis by silver nitrate and other silver preparations is uncertain, since most bacterial cells in cultures of Ps aeruginosa described as silver-resistant are fully sensitive. Experiments in progress, to be reported elsewhere, suggest that dressings soaked in $0.5 \%$ silver nitrate may act as a barrier against contamination of the burn by silverresistant as well as ty silver-sensitive strains.

We are grateful to the Cross Infection Reference Laboratory, Central Public Health Laboratory, Colindale, for serological and phage typing of strains of Ps aeruginosa.

\section{References}

1 Lowbury, E J L, et al, Lancet, 1969, 2, 448.

${ }^{2}$ Lowbury, E J L, et al, Lancet, 1971, 2, 1105.

3 Lowbury, E J L, British Fournal of Hospital Medicine, 1976, 16, 566.

'Cowan, S T, Cowan and Steel's Manual for the Identification of Medical Bacteria, 2nd edn. London, Cambridge University Press, 1975.

${ }^{5}$ Davis, B T, Lilly, H A, and Lowbury, E J L, Fournal of Clinical Pathology, 1969, 22, 634 (and erratum, 23, 177).

${ }^{6}$ Cason, J S, et al, British Medical fournal, 1966, 2, 1288.

${ }^{7}$ Lowbury, E J L, Babb, J R, and Roe, E, Lancet, 1972, 2, 941. 
${ }^{8}$ Bridges, K, and Lowbury, E J L, fournal of Clinical Pathology, 1977, 30, 160.

' Roe, E, Jones, R J, and Lowbury, E J L, Lancet, 1971, 1, 149.

10 Stone, H, in Contemporary Burn Management, ed H C Polk and H H Stone, p 203. Boston, Little Brown, 1971.

11 Bryan, L E, Shahrabadi, M S, and Van den Elzen, H M, Antimicrobial Agents and Chemotherapy, p 191. Washington, American Society for Microbiology, 1974.
12 Kontomichalou, P, Papachristou, E, and Angelatou, F, Antimicrobial Agents and Chemotherapy, p 866. Washington, American Society for Microbiology, 1976.

13 Witchitz, J L, and Chabbert, Y A, Annales de l'Institut Pasteur, 1972, 122, 367.

14 Roe, E, and Lowbury, E J L, fournal of Clinical Pathology, 1972, 25, 176.

(Accepted 20 December 1978)

\title{
Alternative postmeniscectomy regimen
}

\author{
T SERGEANT, A EDWARDS
}

British Medical fournal, 1979, 1, 449-450

\section{Summary and conclusions}

Eighty patients who had undergone routine meniscectomy were divided into two groups for postoperative management. The first 40 patients were treated by the traditional method of a compression bandage with a backsplint, followed by a 10-day stay in hospital. In the other 40 patients a compression bandage was applied, reinforced by a plaster cylinder. Weight-bearing was allowed with the aid of crutches, and patients were discharged after four days in hospital.

Adequate splintage and early ambulation was more convenient and comfortable for postoperative management, and no complications ensued. Less time was needed for physiotherapy, and earlier return to work and resumption of normal activities were made easier.

\section{Introduction}

Meniscectomy is one of the commonest orthopaedic operations, and much has been published on the mechanism of the injury

\section{Coventry and Warwick Hospital, Coventry CV1 4FH}

T SERGEANT, FRCSED, consultant orthopaedic surgeon

Royal Orthopaedic Hospital, Birmingham B31 2AP A EDWARDS, FRCS, senior registrar and the diagnosis of a tear as well as the operative technique. Little attention, however, has been paid to postoperative management. Many orthopaedic surgeons use the traditional method, as outlined by Watson Jones ${ }^{1}$ - namely, a compression bandage with or without a backsplint and early and intensive quadriceps exercises followed by weight-bearing when the sutures are removed on the 10th day. Other workers ${ }^{2} 3$ have advised early mobilisation in a compression bandage, and O'Donoghue $^{4}$ recommended weight-bearing on the second or third day. Early movement, however, is often painful, producing a large effusion and wasting of the quadriceps muscles. ${ }^{5}$ If movement could be prevented by adequate splintage discomfort would be lessened and the patient become ambulant sooner, thus helping to reduce quadriceps wasting.

To test this theory we compared postoperative progress and function in two groups of patients. The first were treated by traditional methods and the second by immobilisation and early weight-bearing.

\section{Patients and methods}

Eighty consecutive patients who had undergone routine meniscectomy for a demonstrable lesion of the meniscus were divided into two groups for postoperative management. All operations were performed by, or under the immediate supervision of, one of us. Patients with other conditions affecting the knee-for example, ligamentous ruptures or bone pathology-were excluded.

Group 1-Forty of the 80 patients were treated by compression bandage and backsplint, and quadriceps exercises were begun on the second postoperative day. Weight-bearing was not permitted until the sutures had been removed. All of these patients remained in hospital for a minimum of 10 days.

Group 2-In the second group of 40 patiencs a compression

TABLE I-Details of patients who had undergone meniscectomy and received either traditional postoperative treatment or were treated by adequate splintage and early mobilisation

\begin{tabular}{|c|c|c|c|c|c|c|c|c|c|c|c|c|c|c|}
\hline & \multirow{2}{*}{ No } & \multirow{2}{*}{$\begin{array}{c}\text { Average } \\
\text { age }\end{array}$} & \multicolumn{2}{|c|}{ Sex } & \multicolumn{2}{|c|}{ Occupation } & \multicolumn{3}{|c|}{ Sporting activities } & \multicolumn{3}{|c|}{ Duration of symptoms } & \multicolumn{2}{|c|}{ Side of injury } \\
\hline & & & $\mathbf{M}$ & $F$ & Light & Heavy & Professional & Amateur & None & $<4$ wks & 4 wks -3 mths & $>3 \mathrm{mths}$ & Medial & Lateral \\
\hline $\begin{array}{l}\text { Traditional management } \\
\text { Splintage and early mobilisation }\end{array}$ & $\begin{array}{l}40 \\
40\end{array}$ & $\begin{array}{l}28 \cdot 2 \\
29 \cdot 9\end{array}$ & $\begin{array}{l}30 \\
32\end{array}$ & $\begin{array}{r}10 \\
8\end{array}$ & $\begin{array}{l}36 \\
35\end{array}$ & $\begin{array}{l}4 \\
5\end{array}$ & $\begin{array}{l}3 \\
5\end{array}$ & $\begin{array}{l}26 \\
25\end{array}$ & $\begin{array}{l}11 \\
10\end{array}$ & $\begin{array}{l}4 \\
6\end{array}$ & $\begin{array}{l}16 \\
16\end{array}$ & $\begin{array}{l}20 \\
18\end{array}$ & $\begin{array}{l}26 \\
29\end{array}$ & 14 \\
\hline
\end{tabular}

TABLE II-Operative findings in patients who underwent meniscectomy and were managed postoperatively either traditionally or by adequate splintage and early mobilisation. Figures are numbers of patients

\begin{tabular}{lcccc}
\hline Type of lesion: & Posterior & Anterior & Bucket-handle & Degenerative \\
\hline $\begin{array}{l}\text { Traditional management } \\
\text { Splintage and early mobilisation }\end{array}$ & 11 & 10 & 14 & 2 \\
3 & 11 & 15 & 3 \\
\hline
\end{tabular}

